\title{
Micromechanics of the internal bond in wood plastic composites: integrating measurement and modeling
}

\author{
Matthew Schwarzkopf ${ }^{1}$ (D) Lech Muszyński ${ }^{2}$ • \\ Chad C. Hammerquist ${ }^{3}$. John A. Nairn ${ }^{4}$
}

Received: 19 August 2016/Published online: 28 June 2017

(C) The Author(s) 2017. This article is an open access publication

\begin{abstract}
In this study, an integrated approach combining experimental measurements and numerical modeling was used for characterization of load transfer in the wood/matrix interface in wood plastic composites (WPCs). The experimental methodology was based on optical measurement of surface displacements and strains in model WPC specimens subjected to tensile loads. The model specimens consisted of thin HDPE films with single embedded wood particles. The optical measurement of surface strains was based on the digital image correlation principle. The material point method was used for morphology-based numerical modeling of the loaded specimens. The exact location and morphology of the embedded particle determined by X-ray computed tomography were used as input for the numerical model. Imperfect interface characteristics, reflecting the efficiency of the load transfer through the interface in the numerical model of the composite, were determined using inverse problem methods. Good agreement was obtained between the simulated and measured strain maps determined on a number of specimens including particles with various orientations to the loading direction using the same values of interface parameters.
\end{abstract}

Matthew Schwarzkopf

matthew.schwarzkopf@iam.upr.si

1 Department of Technology, Andrej Marušič Institute, University of Primorska, Muzejski trg 2, 6000 Koper, Slovenia

2 Department of Wood Science and Engineering, Oregon State University, 106 Richardson Hall, Corvallis, OR 97331, USA

3 Department of Wood Science and Engineering, Oregon State University, 119 Richardson Hall, Corvallis, OR 97331, USA

4 Department of Wood Science and Engineering, Oregon State University, 112 Richardson Hall, Corvallis, OR 97331, USA 


\section{Introduction}

Wood plastic composites (WPCs) are heterogeneous materials comprised of irregular wood particles dispersed in thermoplastic polymer matrices. The matrix is typically high-density polyethylene (HDPE), polypropylene (PP), or poly (vinyl chloride) (PVC). The mechanical properties of such composites are determined by the micromechanics of the internal bonds between wood particles and the matrix. The ability to model and predict this interaction is crucial for designing improved, more efficient composites. This ability relies on a thorough knowledge and ability to predict the effect and efficiency of phase interaction on the bulk properties of the composite. Existing theories and models for short fiber composites allow prediction of composite properties based on the morphology of the composite, mechanical properties of the components, and the properties of the internal bond (Nairn and Shir Mohammadi 2015). However, these theories idealize the internal bond and particle morphology, typically considered to be continuous isotropic solids that are smooth and impermeable. Wood flour particles used in WPCs are generated in hammer mills and attrition mills by physically crushing and tearing larger pieces until the desired size is achieved. Their mechanical properties cannot be automatically assumed to match sound bulk wood tissue, but need to be experimentally characterized. It is unclear whether such idealization can be extended to WPCs where wood particles are anisotropic, porous, permeable, and irregular. In addition, there are many reasons that may negatively affect wood particle/matrix interactions (e.g., chemical incompatibility, compounding method, polymer penetration) and the resulting stress transfer between phases (Simonsen et al. 1997).

While bulk mechanical properties of particulate composites like WPCs can be determined by means of standard test methods, lack of reliable quantitative measurement techniques that would allow analysis of the load transfer between matrix and wood particles in composites at a scale relevant to the size of individual wood particles posed a serious challenge for empirical verification of theoretical predictions and numerical models.

Recently, Schwarzkopf and Muszyński (2015a) proposed an experimental methodology based on optical measurement of surface displacements and strains in thin HDPE film model WPCs subjected to tensile loads. The optical measurement technique based on the digital image correlation (DIC) principle returned full-field surface displacement and strain maps of the specimens without physical contact with the surface. One limitation of the method was that the surface measurements alone could not fully resolve the strain patterns in the 3D matrix neighborhood affected by the wood particle interaction, or the zone of influence (ZOI), beneath the surface. Essentially, the surface measurements reflected the magnitude and trajectories of strains at an arbitrary section of the ZOI determined by the position and depth of the particle beneath the surface, somewhat away from the matrixparticle interface through which the loads are transferred.

A parallel study on micromechanics of adhesive bonds in wood by Kamke et al. (2014) proved that a successful characterization of load transfer is possible by combination of direct measurements of the surface strains with morphology-based 
numerical simulations of deformation in the volume beneath the surface. In that study, material point method (MPM) modeling was coupled with micro-X-ray computed tomography (XCT) and optical measurements based on DIC. XCT data were used as direct morphologic and material input to a micromechanical MPM model of the scanned wood-adhesive interphase. The model was then validated by comparing simulated surface strains with those measured optically on the XCT scanned specimens in the course of non-destructive lap-shear tests. Selected model parameters (local mechanical characteristics of the wood and adhesive, and the wood-adhesive interface) were adjusted until a satisfactory agreement was achieved between the surface strains predicted with model simulations and the strains actually measured on the lap-shear specimens. This approach, based on inverse problem methodology, can be used for many applications in a variety of fields, particularly when there is a level of fundamental compatibility between the full-field optical measurement output and the input to the numerical model of the empirical scene (Muszyński and Launey 2010). While the theory of this approach is pretty intuitive, the practical application requires a close collaboration between the experimentalist and the modeler from the early stage of the project, and an efficient integration of these aspects is challenging.

The objective of this study was to adopt this approach for an efficient integration of optically measured deformations and strains, with predictive MPM numerical modeling tools to characterize the load transfer at the internal bond between the particles of wood flour and a polymer matrix in wood plastic composites.

\section{Materials and methods}

The general approach of this project followed the major steps of the methodology described by Muszyński et al. (2013) and Kamke et al. (2014). Specimen fabrication and testing followed methods described by Schwarzkopf and Muszyński (2015b). The outline of the procedure was as follows: (1) manufacture model WPC specimens; (2) use XCT scans for characterization of the internal morphology of the specimens; (3) perform mechanical tests on the specimens and optically measure the surface displacements and strains; (4) numerically simulate the mechanical test with a morphology-based MPM model; and (5) refine model parameters in an iterative process using inverse problem methods to deduce properties of wood particles and of the particle/matrix interface (Muszyński et al. 2013).

\section{Specimen manufacturing}

The authors were not aware of any viable method for planting individual wood particles in plastic films preserving even rudimentary elements of the compounding process, which is crucial for forming a realistic wood particle/matrix interphase, resembling that in WPCs. Therefore, the approach selected in this study was to compound sparsely filled composites and select specimens with isolated wood particles for testing. 


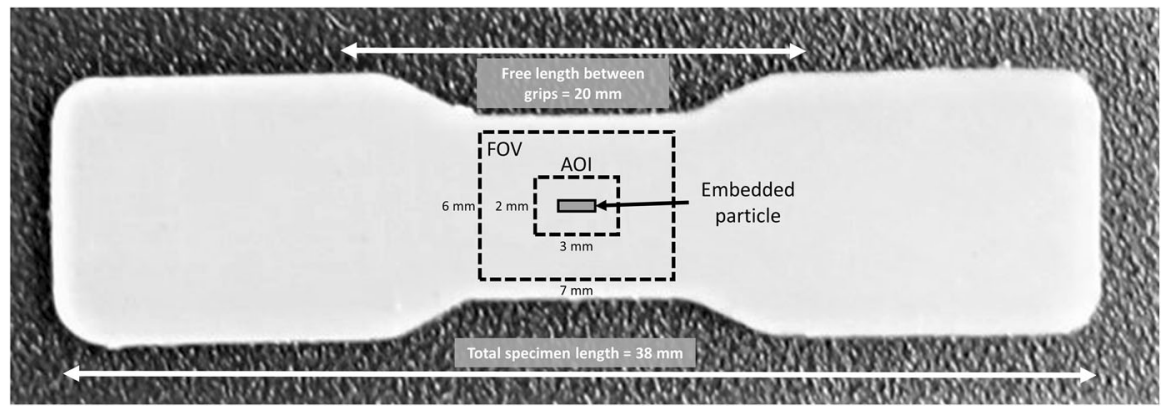

Fig. 1 Test specimen dimensions with optical measurement field of view (FOV) and area of interest (AOI)

The test specimens (Fig. 1) were manufactured from sparsely filled WPC films (less than $0.5 \%$ wood loading). Fortiflex HDPE from Solvay Polymers (Houston, TX, USA) was used for the polymer matrix material. The wood particles used were southern yellow pine (40-mesh, no. 4020) and were obtained from American Wood Fibers (Schofield, WI, USA). The wood particles were dried to a moisture content of $2 \%$ in an oven at $103{ }^{\circ} \mathrm{C}$ in a 30 -mm-thick layer in an aluminum tray. Dried particles were then compounded with HDPE in a counter-rotating, twin screw C.W. Brabender ${ }^{\circledR}$ Instruments, Incorporated (South Hackensack, NJ, USA), IntelliTorque Plasti-Corder ${ }^{\circledR}$ mixer. HDPE (29.93 g) was added to the mixing chamber preheated to $180{ }^{\circ} \mathrm{C}$. The mixing speed was set to $30 \mathrm{rpm}$. When the torque reached a constant level, a pinch of wood particles (approximately $0.1 \mathrm{~g}$ ) was added to the mixing chamber. This small amount of particles led to a sparsely filled composite, from which it was easy to isolate and focus on individual particles ensuring that the analysis of the particle/matrix interaction was not affected by neighboring embedded particles. Once the torque level reached a constant level again, $6 \mathrm{~g}$ of the compounded melt material was transferred to a square mold assembly with hard stops defining internal void dimensions of $102 \mathrm{~mm} \times 102 \mathrm{~mm} \times 0.85 \mathrm{~mm}$ that was preheated to $180{ }^{\circ} \mathrm{C}$ in a small-scale hot press (Carver Inc. Wabash, IN, USA, model Auto M NE, H 3891) to form a film coupon. The mold assembly was held under heat and pressure for $5 \mathrm{~min}$ and then transferred to a cold press until cool (approximately less than $40{ }^{\circ} \mathrm{C}$ ). Test specimens were cut out of the coupon with a steel dog-bone-shaped punch in such a way so that a single wood particle was located in the center of the specimen with approximate orientations of $0^{\circ}, 45^{\circ}$, and $90^{\circ}$ with respect to the principle axis of the specimen.

In addition to the set of specimens with wood particles, a set of reference specimens were made in a similar fashion with small sections of embedded wire instead of wood particles. These reference specimens were intended to mimic the somewhat idealized particle morphology assumed in most existing particle/matrix theories (Tucker and Liang 1999). A more detailed description of the specimen manufacturing process is offered in Schwarzkopf and Muszyński (2015a). 


\section{Characterization of specimen morphology}

In order to account for the effect of the position of the wood particle or embedded wire in the volume beneath the surface, specimens were scanned using a $\mu \mathrm{CT}$ XCT unit (ScanCo, Brüttisellen, Switzerland) at a resolution of $10 \mu \mathrm{m}$ voxel $^{-1}$. Particle sizes and positions within the film were determined manually on the reconstructed scan images using free domain ImageJ software (Rasband 2016). In this process, axial cross sections of the scanned volume were extracted every $40 \mu \mathrm{m}$ along the length of the particle (Fig. 2c).

\section{Mechanical test and surface strain measurements}

Specimens were tested in tension using an Instron ElectroPuls E1000 test machine. The specimen free length of $20 \mathrm{~mm}$ included portions of the tabs on both sides (Fig. 1). Specimens were loaded in displacement mode at a constant rate of $0.5 \mathrm{~mm} /$ min (strain equivalent to $0.02 \mathrm{~mm} / \mathrm{mm}$ per minute) for $2 \mathrm{~min}$. The force was measured using a $\pm 2 \mathrm{kN}$ load cell (Instron 2527 Series Dynacell). During the test,

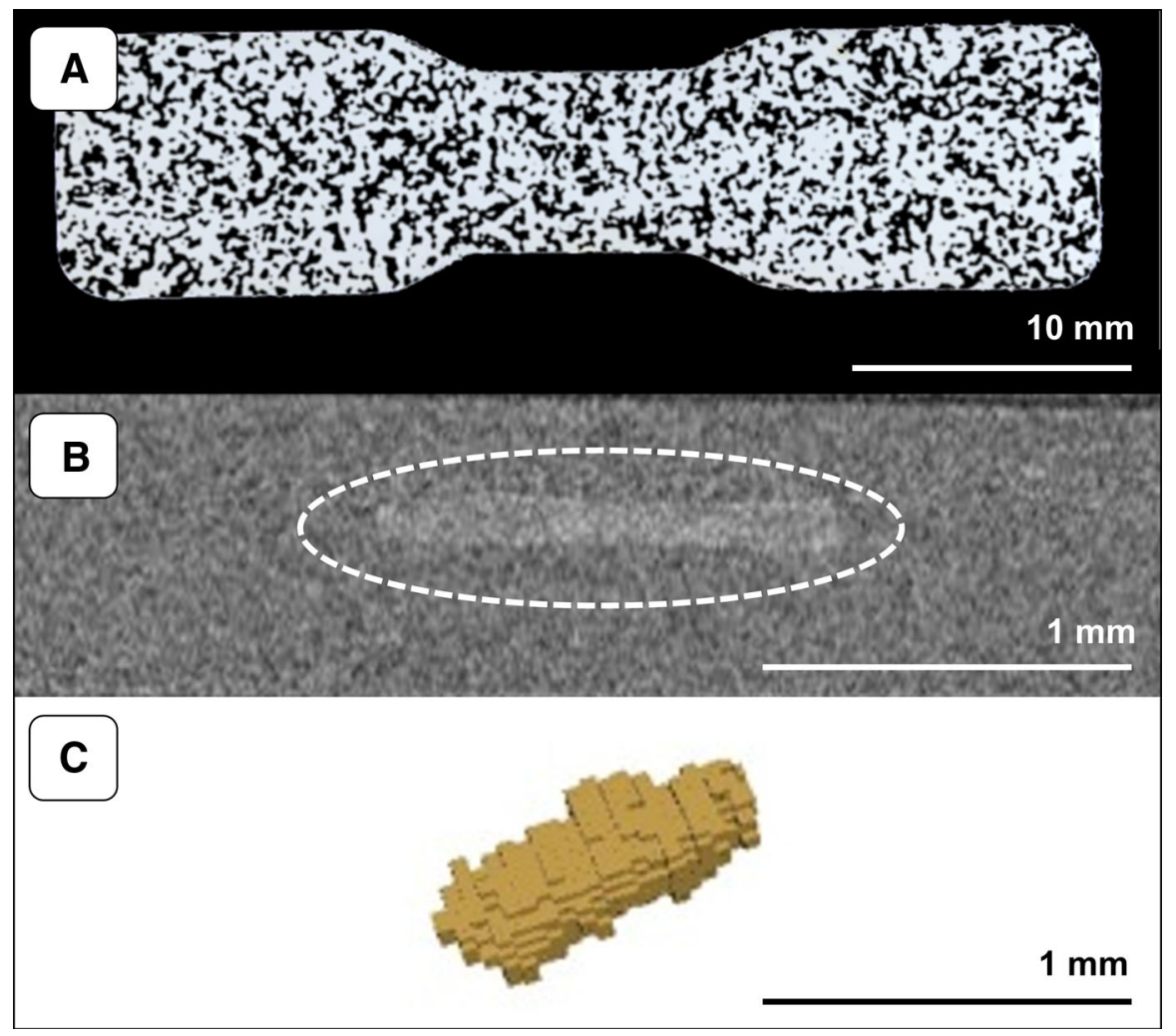

Fig. 2 a WPC specimen with speckle pattern applied. b Cross-sectional view of embedded particle using $\mathrm{X}$-ray computed tomography. c Wood particle geometry determined from XCT scan data 
images of the specimen surface in the immediate neighborhood of the embedded particles (field of view: $7.18 \mathrm{~mm} \times 6.00 \mathrm{~mm}$ ) were recorded every $1 \mathrm{~s}$ for $2 \mathrm{~min}$ with the VIC-Micro 3D ${ }^{\mathrm{TM}}$ system (Correlated Solutions, Inc., Columbia, SC, USA). The working distance from the microscope lens to the specimens was $30 \mathrm{~mm}$.

These images were used to determine surface coordinates, components of displacement vectors, and calculated surface strain tensor components by means of VIC-3D 2012 optical measurement software based on the DIC principle integrated with the optical system. To enhance the DIC analysis, which relies on measuring displacements of unique targets identified on the specimen surface, printer toner was used to apply a fine speckle pattern directly to the light-colored specimen surfaces (Fig. 2a), which provided sufficient contrast with the toner. The pattern was applied by means of air deposition apparatus and fixed on the surfaces by heating in an oven at $103{ }^{\circ} \mathrm{C}$ for $10 \mathrm{~min}$. The technique is described in greater detail in Schwarzkopf and Muszyński (2015a).

The uncertainty of optical measurement is not only a function of the robustness of the DIC algorithm and the attached optical hardware (cameras, lenses, etc.), but is also affected by variables specific to the experimental scene like the quality of the speckle pattern, light, and calibration which at the scale used in this study are much harder to control and assess separately. Therefore, in this project, the accuracy and precision of the optical measurement is performed on the actual test scene of undeformed specimens just before loading. The displacement and strain components for all points on the undeformed specimen are expected to be zero. Thus, simple statistical analysis of nonzero residuals at all points within the area of interest provides us with convenient estimates of systematic bias (average) and random error (standard deviation). These snapshots of undeformed specimens just before the loading allow us, if need be, to analyze the effects of minute differences in speckle pattern distribution between the specimens. This simple routine provides us with an easy metric for the combined uncertainty involved in the optical measurement. This approach was used in the preliminary study to select optimal setup conditions, speckle pattern density, and DIC algorithm parameters: the facet size and the step size. Using a subset facet size of 49 pixels $\times 49$ pixels and a step size of 5 pixels allowed a displacement resolution of $0.038 \mu \mathrm{m} \pm 0.020 \mu \mathrm{m}$ and strain resolution of $50 \mu \mathrm{m} / \mathrm{m} \pm 2.5 \mu \mathrm{m} / \mathrm{m}$.

Axial strain maps (in the direction of loading) were recorded at a nominal stress level of $5 \mathrm{MPa}$ on specimens with particles oriented at an angle of $0^{\circ}, 45^{\circ}$, and $90^{\circ}$ to the direction of load. The maps were then cropped to uniform dimensions and used as reference data for the numerical analysis. The area of interest (AOI) was $3 \mathrm{~mm} \times 3 \mathrm{~mm}$ for specimens with particles inclined at $0^{\circ}$ and $45^{\circ}$ and $3 \mathrm{~mm} \times$ $2 \mathrm{~mm}$ for particles inclined at $90^{\circ}$.

\section{MPM modeling}

The numerical simulations of the tensile tests were carried out using the MPM (Sulsky et al. 1994), which is a particle-based method analogous to finite element analysis (FEA). The particle nature of MPM makes it easier to discretize complex, realistic morphologies (Nairn 2006, 2007; Kamke et al. 2014; Aimene and Nairn 
2015) and also, potentially, allows for improved calculations of interfacial contact and imperfect interfaces (Nairn 2013). Both these advantages were needed for this work.

A custom MPM code called OSParticulas (Nairn 2016) was used to build 3D morphologically accurate numerical models of the regions of interest of individual specimens, which allowed us to run virtual experiments of numerical representations of the specimens used in the physical experiments. In the MPM method, a numerical model can be generated directly from the XCT scans, by turning selected voxels into material points (Kamke et al. 2014). X-ray attenuation of the scanned material reflected by the grayscale intensity of the voxels was used to assign either particle or matrix properties to each material point. In this work, the XCT scans for wood particle and embedded wire specimens had a voxel size of $10 \mu \mathrm{m} \times 10 \mu \mathrm{m} \times$ $40 \mu \mathrm{m}$ (the lower dimension was the axial direction of $0^{\circ}$ particles). These scans were input to MPM calculations, which were run with a particle size of $40 \mu \mathrm{m} \times$ $40 \mu \mathrm{m} \times 40 \mu \mathrm{m}$. Each simulation was comprised of about 1.2 million material points and took about $18 \mathrm{~h}$. Without higher-resolution XCT scans, it had to be assumed that the available resolution was sufficient for accurate numerical modeling. The use of much higher resolution would likely be impractical due to long simulations and the need for multiple simulations to solve inverse problems.

The HDPE matrix was modeled as a small-strain, isotropic material (with one elastic modulus $E$ and one Poisson's ratio $v$ ) with plastic yielding and hardening. The plastic yielding was modeled by standard $J_{2}$ plasticity methods (Simo and Hughes 1998) and used the hardening law:

$$
\sigma_{\mathrm{Y}}=\sigma_{\mathrm{Y} 0}\left(1+K \varepsilon_{\mathrm{p}}\right)^{n}
$$

where $\sigma_{\mathrm{Y}}$ is the current yield stress, $\sigma_{\mathrm{YO}}$ is the initial yield stress, $\varepsilon_{\mathrm{p}}$ is the cumulative plastic strain, and $K$ and $n$ are dimensionless material hardening parameters. HDPE parameters were determined by fitting stress-strain results for reference pure HDPE specimens (which could be done on actual pure specimens or by looking at DIC strains far from a single isolated particle in a large specimen). $E$ was determined from the initial slope; Poisson's ratio, which had little effect, was fixed to $v=0.33 ; \sigma_{\mathrm{YO}}$ was determined from onset on nonlinearity; and $K$ and $n$ were manually varied to fit the nonlinear stress-strain curve after yielding. The determined HDPE properties are given in Table 1. For these short-term simulations, it was assumed that viscoelasticity effects were negligible or that all nonlinearity could be attributed to plasticity. It is noted, however, that for monotonic loading, it makes no difference whether nonlinearities are modeled by plasticity or by viscoelasticity as long as the constitutive law recovers the correct loading response.

The wood was modeled using orthotropic material properties of solid wood. The properties are listed in Table 1, and the long axis of the particle was assumed to be the longitudinal direction of the wood. The properties of wood particles are unknown and likely affected by extraction method and moisture content. This work used low-end properties of clear southern yellow pine at $12 \%$ moisture content (Forest Products Laboratory 2010). Future modeling could either deduce particle properties by inverse methods or try to measure them at appropriate particle 
Table 1 Mechanical properties used for HDPE matrix, wood particles, and copper wire in the MPM numerical modeling

\begin{tabular}{llll}
\hline Property & HDPE & Wood & Copper \\
\hline$E\left(\right.$ or $\left.E_{L}\right)(\mathrm{MPa})$ & 1013 & 9000 & 120,000 \\
$E_{R}$ and $E_{T}(\mathrm{MPa})$ & & 600 & \\
$G_{\mathrm{LR}}$ and $G_{\mathrm{LT}}(\mathrm{MPa})$ & & 700 & \\
$G_{\mathrm{RT}}(\mathrm{MPa})$ & & 108 & \\
$v\left(\right.$ or $\left.v_{\mathrm{RT}}\right)$ & 0.33 & 0.709 & 0.34 \\
$v_{\mathrm{LR}}$ and $v_{\mathrm{LT}}$ & & 0.021 & \\
$\sigma_{\mathrm{Y} 0}\left(\right.$ or $\left.\sigma_{\mathrm{Y}, \mathrm{LL}}\right)(\mathrm{MPa})$ & 8 & $\infty$ & \\
$\sigma_{\mathrm{Y}, \mathrm{RR}}$ and $\sigma_{\mathrm{Y}, \mathrm{TT}}(\mathrm{MPa})$ & & 5 & \\
$\tau_{\mathrm{Y}, \mathrm{LR}}$ and $\tau_{\mathrm{Y}, \mathrm{LT}}(\mathrm{MPa})$ & & 6 & \\
$\tau_{\mathrm{Y}, \mathrm{RT}}(\mathrm{MPa})$ & & 2.5 & \\
$K$ & 8.49 & 120 & \\
$n$ & 0.413 & 4 & \\
$\rho\left(\mathrm{g} / \mathrm{cm}^{3}\right)$ & 1.0 & 0.39 & 8.96 \\
\hline
\end{tabular}

moisture content. To account for possible wood failure during loading, wood failure was modeled using the (Hill 1948) anisotropic failure criterion:

$$
\sqrt{F\left(\sigma_{x x}-\sigma_{y y}\right)^{2}+G\left(\sigma_{x x}-\sigma_{z z}\right)^{2}+H\left(\sigma_{y y}-\sigma_{x x}\right)^{2}+\frac{\tau_{y z}^{2}}{\tau_{Y, y z}^{2}}+\frac{\tau_{x x}^{2}}{\tau_{Y, x z}^{2}}+\frac{\tau_{x y}^{2}}{\tau_{Y, x y}^{2}}}=1+K \varepsilon_{\mathrm{p}}^{n}
$$

where

$$
\begin{aligned}
& F=\frac{1}{2}\left(\frac{1}{\sigma_{Y, y y}^{2}}+\frac{1}{\sigma_{Y, z z}^{2}}-\frac{1}{\sigma_{Y, x x}^{2}}\right) G=\frac{1}{2}\left(\frac{1}{\sigma_{Y, x x}^{2}}+\frac{1}{\sigma_{Y, z z}^{2}}-\frac{1}{\sigma_{Y, y y}^{2}}\right) \\
& H=\frac{1}{2}\left(\frac{1}{\sigma_{Y, x x}^{2}}+\frac{1}{\sigma_{Y, y y}^{2}}-\frac{1}{\sigma_{Y, z z}^{2}}\right)
\end{aligned}
$$

here $\sigma_{i i}$ and $\tau_{i j}$ are current stresses, $\sigma_{Y, i i}$ and $\tau_{Y, i j}$ are material yield strengths for loading in one direction, $\varepsilon_{\mathrm{p}}$ is cumulative plastic strain, and $K$ and $n$ are material hardening parameters for wood. The yield strengths for southern yellow pine wood, which are based on failure loads in the three anatomical directions (Bodig and Jayne 1982; Forest Products Laboratory 2010), are given in Table 1. Although anisotropic wood failure was modeled, little or no plastic deformation was found in the wood particles for these low-strain experiments. The anisotropic failure modeling may be more important in future projects.

Finally, the copper wire was treated as a simple isotropic linear elastic material with properties (Ashby 2011) listed in Table 1.

To complete the model, the wood/HDPE or copper/HDPE interfaces were modeled as imperfect interfaces. The imperfect interface model was derived from the Hashin $(1990,1991)$ analysis for imperfect interfaces in composites, in which 
the normal and shear tractions at the interface $\left(\sigma_{n}\right.$ and $\left.\tau_{t}\right)$ were assumed to be linearly related to a displacement discontinuity between the phases by:

$$
\sigma_{\mathrm{n}}=D_{\mathrm{n}}\left[u_{\mathrm{n}}\right] \quad \text { and } \quad \tau_{\mathrm{t}}=D_{\mathrm{t}}\left[u_{\mathrm{t}}\right]
$$

where $\left[u_{\mathrm{n}}\right]$ and $\left[u_{\mathrm{t}}\right]$ are displacement discontinuities in the normal and tangential directions and $D_{\mathrm{n}}$ and $D_{\mathrm{t}}$ are interphase parameters that describe the quality of the interface. When $D_{\mathrm{n}}=D_{\mathrm{t}}=0$, the tractions on the interface are zero, which models a debonded interface. When $D_{\mathrm{n}}=D_{\mathrm{t}}=\infty$, the discontinuities must be zero, which models a perfect interface. All other values model an imperfect interface with higher values of $D_{\mathrm{n}}$ and $D_{\mathrm{t}}$ reflecting a higher-quality interface. Imperfect interfaces were included in these MPM simulations using methods described in Nairn (2013).

The Hashin (1990, 1991) imperfect interface analysis is based on interfacial stiffness and meant to apply up to the point of debonding or failure. To model strength as well, the relations in Eq. (4) could be modified to have a maximum normal or shear strength. In other words, interfacial failure would have $D_{\mathrm{n}}$ and $D_{\mathrm{t}}$ as functions of displacement discontinuities (possibly being nonlinear) and drop to zero (i.e., convert to a debonded interface) after failure. These experimental and numerical results had no evidence of significant debonding. This work thus focused on interfacial stiffness and avoided introduction of additional interfacial strength parameters.

Finally, to solve the approximate inverse problems, MPM simulations were performed for the same nominal stress level at which the surface strain maps were measured and MPM simulations were done on specimen morphologies derived from $\mathrm{XCT}$ data. In principle, by comparing simulated results to experimental results as a function of all input parameters, one could determine those parameters. In practice, this can be done only for a limited number of input parameters. One way of reducing the amount of computation is to exclude parameters to which the model is less sensitive or focusing on least known parameters, for which good estimates are not available. In this study, to simplify the analysis, the unknowns were reduced to a single property or one interface parameter $D=D_{\mathrm{n}}=D_{\mathrm{t}}$, which was then determined by inverse methods of matching predictions to experiments.

To compare the simulated and measured strains, the axial strain results from the MPM simulations at the surface of the model and the surface strains actually measured on the specimens were mapped to the same spatial grid (shifting MPM results as needed to overlap experimental features). To determine the accuracy of the model simulation, the difference between the observed and simulated strains was calculated and used to quantify errors. The differences in surface axial strain between the simulation and measurement were summarized using mean absolute error (MAE) between the experimental data and the simulations determined for each point $i$,

$$
\text { MAE }=\frac{1}{n} \sum_{i=1}^{n}\left|f_{i}-y_{1}\right|
$$

where $f_{i}$ is the simulation value, and $y_{i}$ is the measured value. To give a more absolute measure, a relative measure or symmetric mean absolute percent error (SMAPE): 


$$
\mathrm{SMAPE}=\frac{100}{n} \sum_{i=1}^{n} \frac{\left|f_{i}-y_{1}\right|}{\frac{1}{2}\left(\left|f_{i}\right|+\left|y_{1}\right|\right)}
$$

was also used to summarize agreement between experiments and models. Local minimum in these values with respect to the interface parameter can give an estimate of interfacial properties.

These interface properties are difficult to verify independently. However, some degree of validation may be achieved by comparing the results obtained for the wire reference with theoretical results obtained using one of the short fiber theories and/ or by comparing interface property values determined on different specimens with wood particles used in this study.

\section{Results and discussion}

The initial results presented here are proof of concept for an integrated procedure as a tool for characterizing load transfer between particles and matrix in WPCs expressed through the interphase integrity parameter $D$. First, the method was validated by using a model system with a copper wire embedded in HDPE instead of a wood particle. This model material was used because the properties of the component phases were determined with little uncertainty and because the phases were certain not to mix at the interface, which left the interfacial properties as the only unknown material parameter in the composite.

\section{Composites with wire particles}

Figure 3 illustrates the iterative process of refining the model parameters to fit the measured surface strains for a short copper wire piece embedded in HPDE at an approximately $0^{\circ}$ orientation, under tension at a stress of $5 \mathrm{MPa}$. The left column of Fig. 3 shows experimental axial strain data obtained from optical measurements (DIC) (these are reproduced in each row of the chart for comparison). The middle column shows simulated surface strains from 3D MPM calculations for various values of interface parameter $D$. The right column shows difference in strains between experimental and numerical calculations. The magnitude of absolute strain is defined by the scale bar from 0.000 to 0.008 .

The experimental results show low axial strain near the center of the copper wire. At either end of the embedded particle, high strain concentrations in the matrix are seen. This behavior is the expected behavior arising from mechanics of load transfer from compliant matrix to stiff wire. All observations are consistent with measured and theoretical descriptions of load transfer in short fiber composites (Cox 1952; Clyne 1989; Nairn 2004; Sretenovic et al. 2006; Schwarzkopf and Muszyński 2015a).

The MPM results in the middle show numerical predictions with $D=\infty$ (representing a perfect interface) and then for decreasing values of $D=10,000$, 5000 , and $1000 \mathrm{MPa} / \mathrm{mm}$ representing increasing level of imperfection of the 


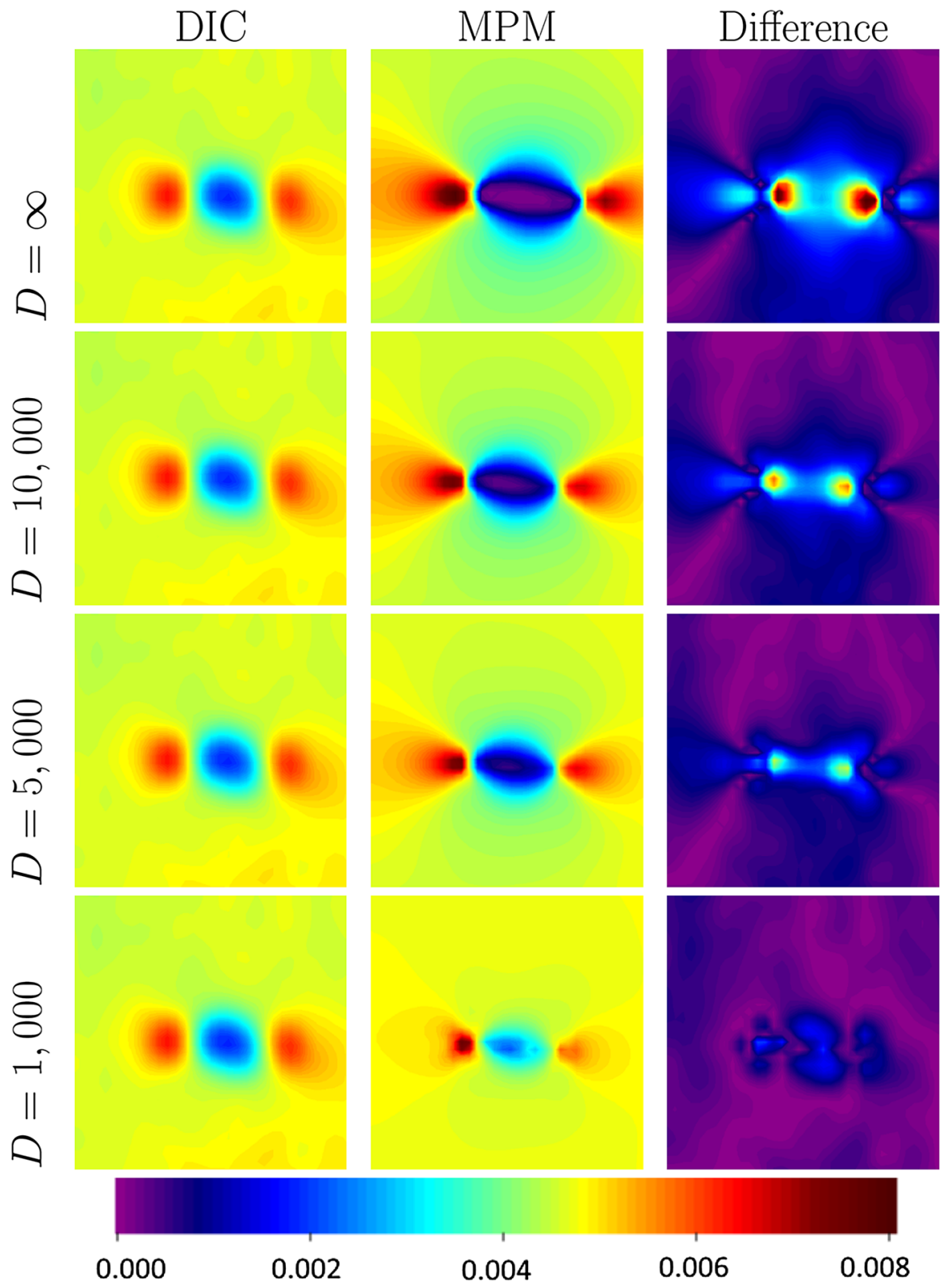

Fig. 3 Axial strain plots of copper wire/HDPE composite. Plots in the first column are optical measurements compared with MPM simulation results in the second column. The difference in strain between the two is plotted in the third column. From top to bottom, the interface parameter used in the simulation is decreasing

internal bond. The perfect interface simulation $(D=\infty)$ shows lower strain in the fiber and higher stress concentration in the matrix. Both of these differences indicate better stress transfer in the simulation than in the experiments (Nairn 2004). With 
the decreasing value of the interface parameter reflecting less perfect stress transfer between the wire and the matrix, the simulated surface strains gradually became closer to those measured experimentally.

To visualize the difference between simulation and experiments, plots mapping the magnitude of the net difference were added in the right column. A perfect agreement would be seen as a 0.000 difference across the entire area in the right column. For $D=1000$, regions of disagreement were lowest with the maximum difference being about 0.002 strain $(20,000 \mu \mathrm{m} / \mathrm{m})$. This is well within the DIC measurement resolution of $50 \mu \mathrm{m} / \mathrm{m} \pm 2.5 \mu \mathrm{m} / \mathrm{m}$.

The SMAPE average calculated with Eq. (6) for datasets in Fig. 3 and for some additional values of interface parameter $D$ is plotted in Fig. 4. The smoothed curve shows a clear minimum around $D$ value between 1000 and $2000 \mathrm{MPa} / \mathrm{mm}$. Thus, the inverse methodology has determined interfacial properties through coupling of experimental results and numerical simulation on the same structure.

The hypothesis is that the $0^{\circ}$ copper wire experiments correctly measured the quality of the copper/HDPE interface. Because interfacial properties should not depend on wire orientation, the same value of $D$ should similarly give good simulations results for wires at $45^{\circ}$ and $90^{\circ}$ to the loading direction. This hypothesis was tested by comparing experiments to simulations for all wire orientations, as illustrated in Fig. 5 (using the same outline as in Fig. 3). All difference plots (right column) show good overall agreement between the model and the experimental measurements.

In Fig. 6, the SMAPE error estimates for all three angles are compared. The errors for $45^{\circ}$ and $90^{\circ}$ were slightly higher, but still reasonably low. One could argue that the different interfacial stress states seen for different orientations might influence the interfacial slip (e.g., ratio on normal and tangential interfacial stresses reflected in two interface parameters, $D_{\mathrm{n}}$ and $D_{\mathrm{t}}$, instead of the single parameter used here). The error could certainly be reduced by adjusting these two interface parameters independently

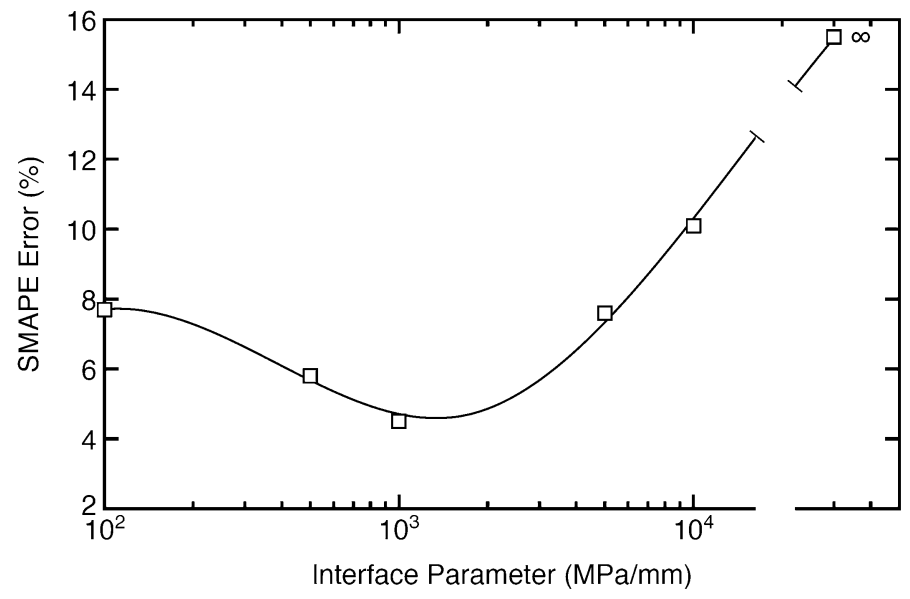

Fig. 4 SMAPE error estimate for the copper wire/HDPE simulations along with some additional values of interface parameter $D$. The line is a smoothing curve through error estimates. The perfect interface result is marked with $\infty$ and plotted on the right after a gap in the plot 


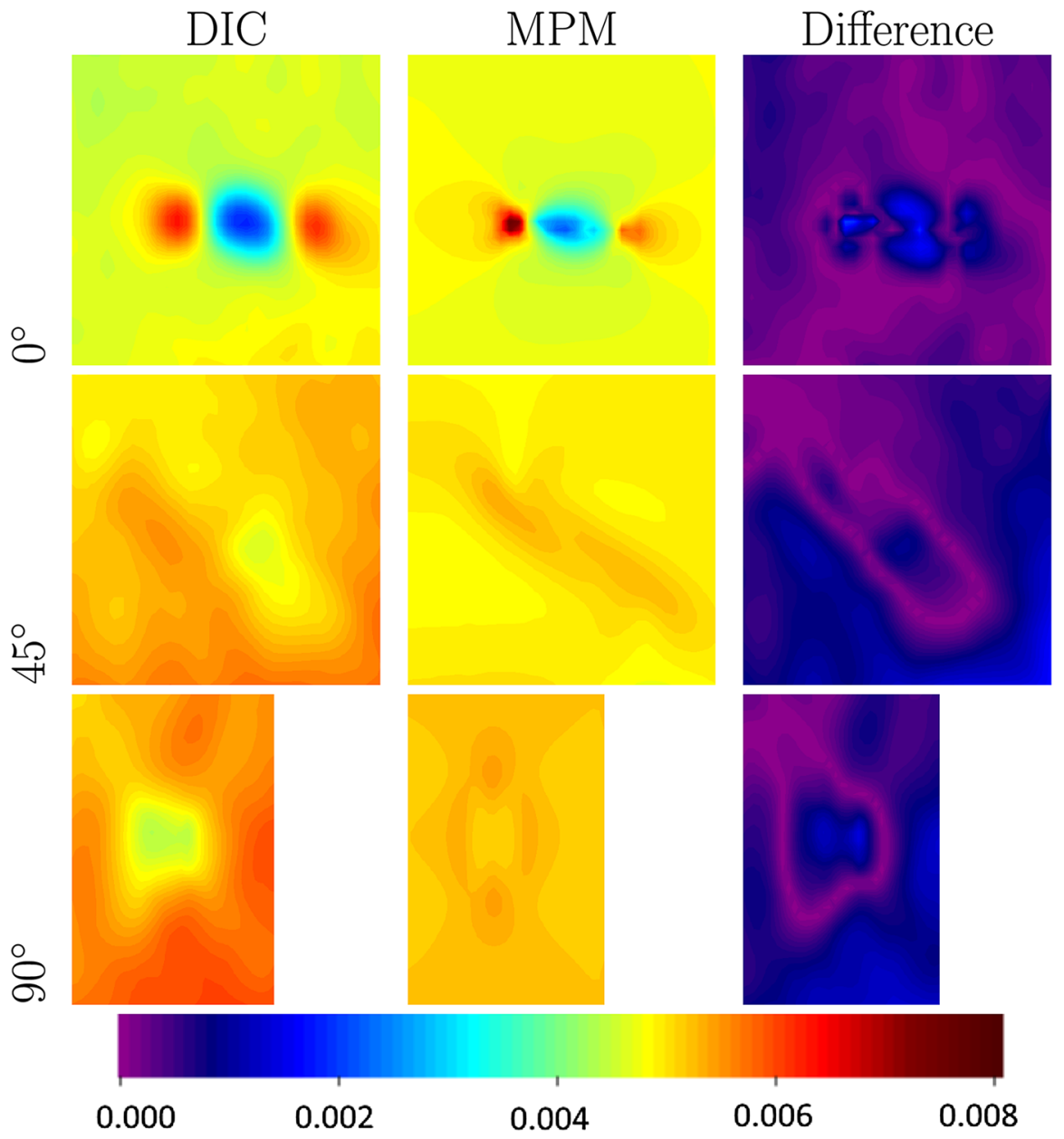

Fig. 5 Axial strain plots from optical measurements of embedded wire particles at varying orientations. The first column is compared with MPM simulation results in the second column. The difference in strain between the two is plotted in the third column. The interface parameter used in the simulations was $D=1000 \mathrm{MPa} / \mathrm{mm}$

(two parameters will always reduce errors compared to using a single parameter). Instead it was chosen to keep them the same to see whether a single interfacial value can predict all orientations, and a single parameter worked reasonably well. It would likely require additional experiments under different stress states before one could determine two parameters with high confidence.

\section{Composites with wood particles}

The next comparison is focused on the strain maps obtained from simulations and experimental measurements for specimens with embedded wood particles oriented 


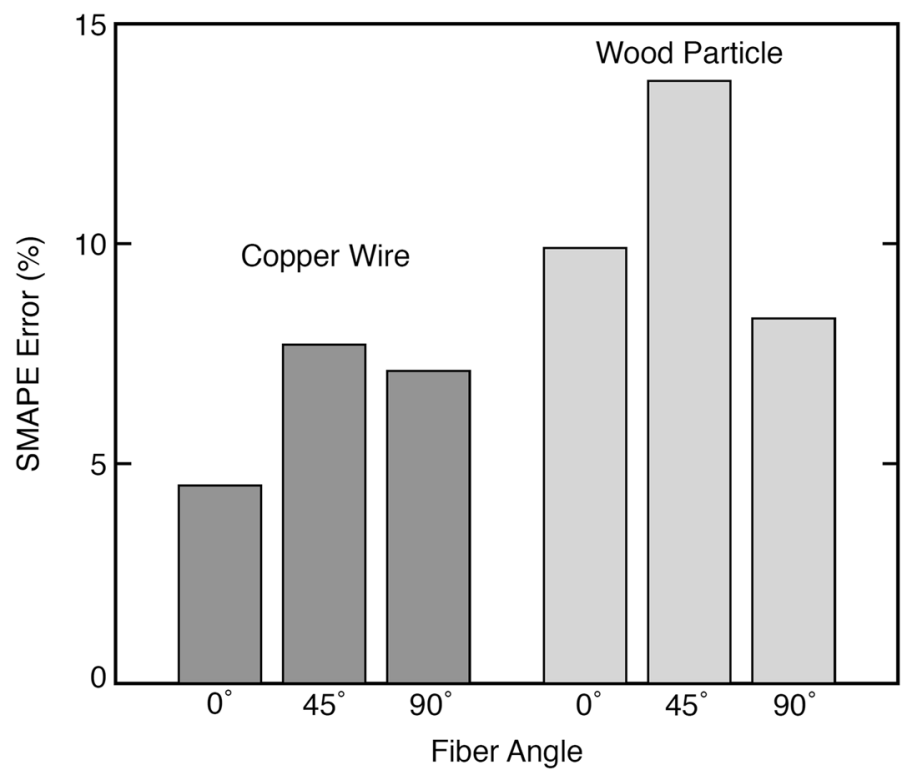

Fig. 6 SMAPE error estimate for the copper wire/HDPE simulations and for wood particle/HDPE simulations as a function of wire or particle angle with respect to the loading direction. The interface parameter for copper wire/HDPE was $D=1000 \mathrm{MPa} / \mathrm{mm}$ and for wood particle/HDPE simulations, $D=\infty$

at $0^{\circ}$ to the loading direction. Figure 2 shows the specimen, and Fig. 2c shows MPM discretization of the particle derived from XCT experiments. The MPM model shows only the wood particle material points, while the material points for the matrix have been removed for clarity. The particle had a much rougher surface than the copper wire, but, by using XCT data, the simulations can model the roughness and can match roughness of simulated particles to the roughness of the experimental specimen.

The SMAPE error metric for the wood particle specimen as a function of interface parameter $D$ is shown in Fig. 7. The error drops as $D$ increases, suggesting that the load transfer through the interface is nearly perfect. The possibility of a minimum at higher $D$ values is difficult to verify because imperfect interface models become less stable for large $D$ (Nairn 2013). This difficulty is easily avoided by switching the analysis to the perfect interface model $(D=\infty)$, which is stable because it simply omits the need to evaluate interfacial tractions. In brief, the wood/HDPE interface appears to be excellent. This finding is contrary to the common belief that the polar wood component and the nonpolar thermoplastic matrix are chemically incompatible and so only weak physical bonding is expected to occur between the wood cell wall and unmodified HDPE. It is believed that this strong interface is due to the rough surface of the wood particle allowing mechanical interlocking in the interphase (Wang 2007). 


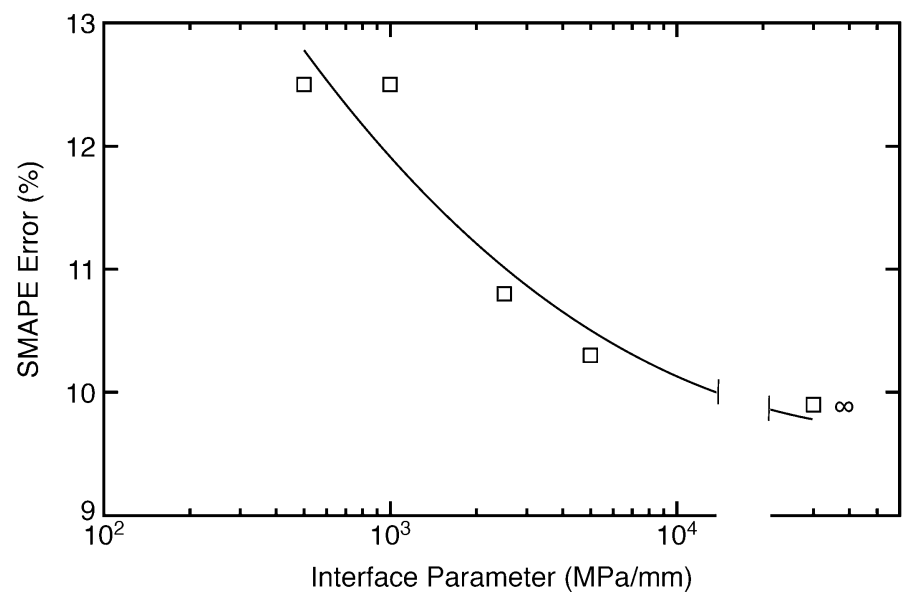

Fig. 7 SMAPE error estimate for the wood particle/HDPE simulations as a function of interface parameter $D$. The line is a smoothing curve through error estimates. The perfect interface result is marked with $\infty$ and plotted on the right after a gap in the plot

\section{General discussion}

Another difficulty in interpreting experiments with embedded wood particles compared to experiments with embedded copper was that the mechanical properties of hammer milled wood particles were estimated with greater uncertainty due to lack of reliable data. The wood properties used for numerical simulations (Table 1) were set at the low end of values quoted for clear southern yellow pine and for simplicity used $E_{R}=E_{T}$ and $G_{\mathrm{LR}}=G_{\mathrm{LT}}$ (they are similar). The material properties are still, however, orthotropic because of low $G_{\mathrm{RT}}$. Although it is possible the properties of particles would be reduced compared to clear wood due to hammer milling used to extract them, the authors did not have properties specific for particles. The choice for wood properties might have affected the results for $D$, which was assumed to be the only needed variable in the numerical analysis. The authors tried scaling down wood properties by various factors and did not find significant differences in the overall conclusions. All simulations shown here thus used the full properties in Table 1. A better approach would be to deduce both the mechanical properties of wood and the imperfect interface factor by inverse problem methods. This more advanced approach would require coupled analysis of greater number of experimental specimens, which is beyond the scope of this proofof-concept study.

Instead, consistency of the outcome obtained for specimens with particles aligned with the direction of load (perfect internal bond, or $D=\infty$ ) was tested by comparing the simulations obtained for particles oriented at $45^{\circ}$ and $90^{\circ}$ to the loading direction with the respective experimental results using the same perfect interface assumption. The strain fields for all orientation angles are compared in Fig. 8. The comparisons are reasonably accurate, although the maximum difference in some small regions reached about 0.003 , which is somewhat larger than for the 


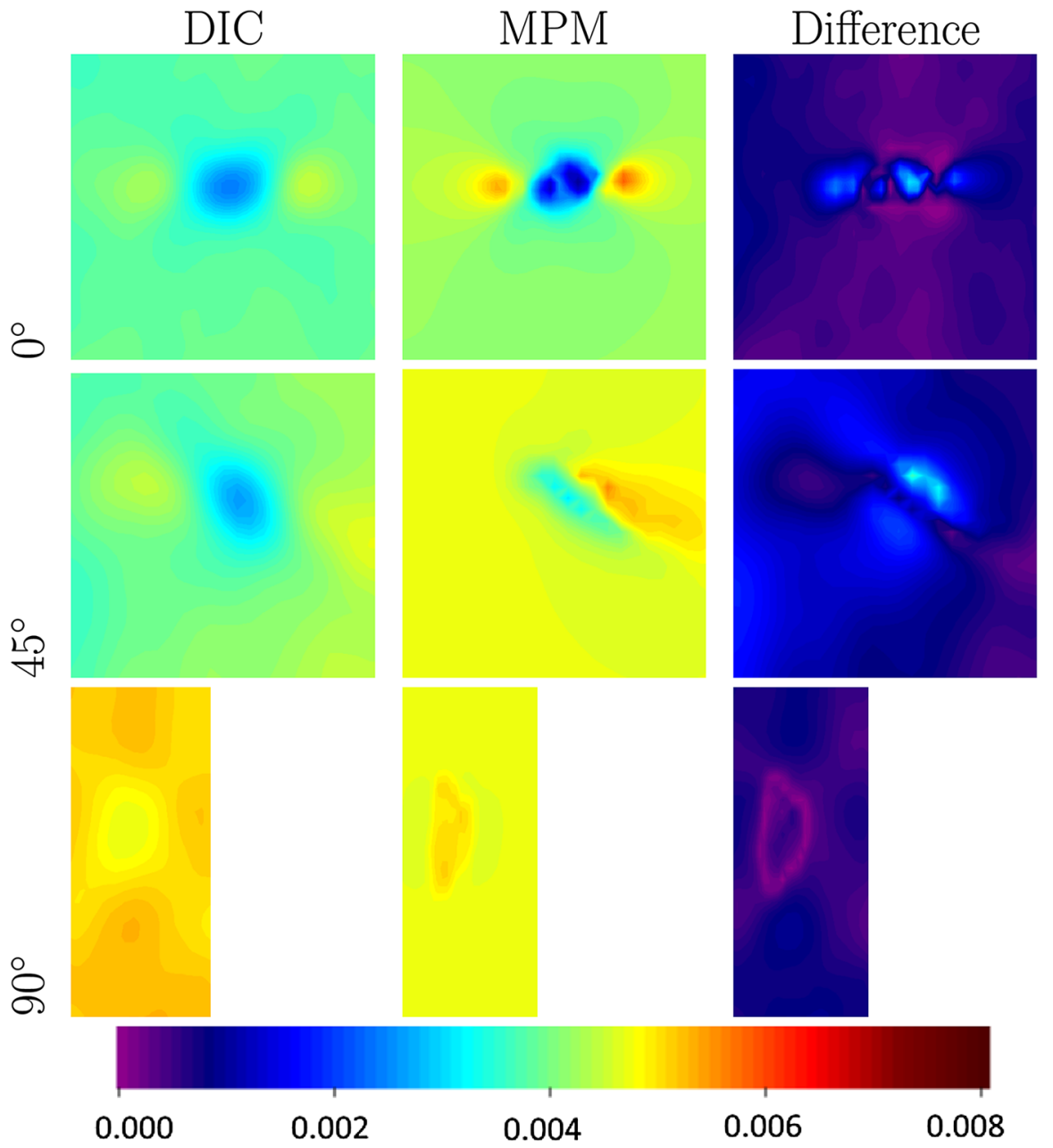

Fig. 8 Axial strain plots from optical measurements of embedded wood particles at varying orientations. The first column is compared with MPM simulation results in the second column. The difference in strain between the two is plotted in the third column

copper experiments. The SMAPE error estimates for different wood particle orientations are given in Fig. 7. Again, the errors are higher than for copper wire, but still in the range of $8 \%$ to $14 \%$, and the same value of $D=\infty$ fits all experiments with a similar magnitude of error.

\section{Conclusion}

An integrated method was presented for coupling experimental measurements of strain fields on the surface of wood particle composites with 3D numerical simulations of the strain fields in the specimen volume to assess particle/matrix load 
transfer and interfacial properties of the composite. The analysis was performed at a spatial scale that is relevant to the wood particle/matrix interactions. The numerical model was based on morphological input data from XCT scans. These scans were needed to properly reflect the particle position within the specimens and to capture effects of surface roughness of wood particles.

Inverse problem methods were used to determine one input parameter-an imperfect interface value $D(\mathrm{MPa} / \mathrm{mm})$ - used in the model to characterize the efficiency of load transfer between the particle and the matrix. The value of this parameter was varied until good agreement was obtained between the simulated and measured strain maps determined on a number of specimens including particles with various orientations to the loading direction using the same values of interface parameters. The other uncertain parameters were assumed as known.

A relatively low value of this parameter $(D=1000 \mathrm{MPa} / \mathrm{mm})$ was determined for reference copper wire/HDPE composites meant to simulate the idealized short fiber/matrix interface. By contrast, an effectively perfect interface parameter $(D=\infty)$ was found to provide simulations closest to the actual measurements for wood/HDPE composite samples. This high load transfer efficiency in wood/HDPE composite samples might be due to mechanical interlocking in the porous wood/ polymer interphase observed in other studies.

In future work, the analysis may be fine-tuned by increasing the number of unknown parameters to be determined in the inverse problem method (e.g., the mechanical properties of wood particles). This work, however, will require a greater number of input experiments, different loading modes, multiple particle interactions, and greater computing power.

Acknowledgements The authors gratefully acknowledge: Urszula Iwaniec and the micro-XCT laboratory at OSU for conducting the XCT scans, the USDA National Research Initiative Grant Program Award No. 2008-35504-19227, the National Science Foundation grant CMMI 1161305, and the European Commission for financial support through the project InnoRenew CoE, Grant Agreement \#664331 under the Horizon2020 Widespread-2015 program.

Open Access This article is distributed under the terms of the Creative Commons Attribution 4.0 International License (http://creativecommons.org/licenses/by/4.0/), which permits unrestricted use, distribution, and reproduction in any medium, provided you give appropriate credit to the original author(s) and the source, provide a link to the Creative Commons license, and indicate if changes were made.

\section{References}

Aimene YE, Nairn JA (2015) Simulation of transverse wood compression using a large-deformation, hyperelastic-plastic material model. Wood Sci Technol 49:21-39

Ashby MF (2011) Materials selection in mechanical design, 4th edn. Elsevier, Kidlington

Bodig J, Jayne BA (1982) Mechanics of wood and wood composites. Van Nostrand Reinhold, New York Clyne TW (1989) A simple development of the shear lag theory appropriate for composites with a relatively small modulus mismatch. Mater Sci Eng A 122:183-192

Cox HL (1952) The elasticity and strength of paper and other fibrous materials. Br J Appl Phys 3:72-79 
Forest Products Laboratory (2010) Wood handbook: wood as an engineering material. General Technical Report FPL-GTR-190. Madison, WI: U.S. Department of Agriculture, Forest Service, Forest Products Laboratory

Hashin Z (1990) Thermoelastic properties of fiber composites with imperfect interface. Mech Mater 8:333-348

Hashin Z (1991) Thermoelastic properties of particulate composites with imperfect interface. J Mech Phys Solids 39:745-762

Hill R (1948) A theory of the yielding and plastic flow of aniostropic metals. Proc R Soc Lond Ser A Math Phys Sci 193:281-297

Kamke F, Nairn JA, Muszyński L, Paris JL, Schwarzkopf M, Xiao X (2014) Methodology for micromechanical analysis of wood adhesive bonds using X-ray computed tomography and numerical modeling. Wood Fiber Sci 46:15-28

Muszyński L, Launey ME (2010) Advanced imaging techniques in wood-based panels research. In: Wood-based panels: an introduction for specialists. State-of-the-art in wood-based panels research, COST action E49, pp 177-201

Muszyński L, Kamke FA, Nairn JA, Schwarzkopf M, Paris J (2013) Integrated method for multiscale/multi-modal investigation of micro-mechanical wood-adhesive interaction. In: International conference on wood adhesives, Toronto, Ontario, Canada, p 17

Nairn JA (2004) Generalized shear-lag analysis including imperfect interfaces. Adv Compos Lett 13:263-274

Nairn JA (2006) Numerical simulations of transverse compression and densification in wood. Wood Fiber Sci 38:576-591

Nairn JA (2007) Material point method simulations of transverse fracture in wood with realistic morphologies. Holzforschung 61:375-381

Nairn JA (2013) Modeling of imperfect interfaces in the material point method using multimaterial methods. Comput Model Eng Sci 92:271-299

Nairn JA (2016) Material point method (Nairn MPM) open-source software. http://osupdocs.forestry. oregonstate.edu/index.php/Main_Page

Nairn JA, Shir Mohammadi M (2015) Numerical and analytical modeling of aligned short fiber composites including imperfect interfaces. Compos Part A 77:26-36

Rasband W (2016) ImageJ. U.S. National Institutes of Health, Bethesda, MD, USA. http://imagej.nih.gov/ ij

Schwarzkopf M, Muszyński L (2015a) Strain distribution and load transfer in the polymer-wood particle bond in wood plastic composites. Holzforschung 69:53-60

Schwarzkopf M, Muszyński L (2015b) Stereomicroscopic optical method for the assessment of load transfer patterns across the wood-adhesive bond interphase. Holzforschung 69:653-660

Simo JC, Hughes TJR (1998) Computational inelasticity. Springer, New York

Simonsen J, Hong ZQ, Rials TG (1997) The properties of the wood-polystyrene interphase determined by inverse gas chromatography. Wood Fiber Sci 29:75-84

Sretenovic A, Müller U, Gindl W (2006) Mechanism of stress transfer in a single wood fibre-LDPE composite by means of electronic laser speckle interferometry. Compos Part A 37:1406-1412

Sulsky D, Chen Z, Schreyer HL (1994) A particle method for history-dependent materials. Comput Methods Appl Mech Eng 118:179-186

Tucker CL III, Liang E (1999) Stiffness predictions for unidirectional short-fiber composites: review and evaluation. Compos Sci Technol 59:655-671

Wang Y (2007) Morphological characterization of wood plastic composite (WPC) with advanced imaging tools: developing methodologies for reliable phase and internal damage characterization. Thesis. Oregon State University, Corvallis 